\title{
ACTIVE DAMPING USING DISTRIBUTED ANISOTROPIC ACTUATORS
}

\author{
Noah H. Schiller \\ NASA Langley Research Center \\ Hampton, Virginia, USA
}

\author{
Juan D. Quinones \\ University of Puerto Rico \\ Mayaguez, Puerto Rico, USA
}

\author{
Randolph H. Cabell \\ NASA Langley Research Center \\ Hampton, Virginia, USA \\ Nathan C. Wier \\ Michigan Technological University \\ Houghton, Michigan, USA
}

\begin{abstract}
A helicopter structure experiences substantial highfrequency mechanical excitation from powertrain components such as gearboxes and drive shafts. The resulting structureborne vibration excites the windows which then radiate sound into the passenger cabin. In many cases the radiated sound power can be reduced by adding damping. This can be accomplished using passive or active approaches. Passive treatments such as constrained layer damping tend to reduce window transparency. Therefore this paper focuses on an active approach utilizing compact decentralized control units distributed around the perimeter of the window. Each control unit consists of a triangularly shaped piezoelectric actuator, a miniature accelerometer, and analog electronics. Earlier work has shown that this type of system can increase damping up to approximately $1 \mathrm{kHz}$. However at higher frequencies the mismatch between the distributed actuator and the point sensor caused control spillover.

This paper describes new anisotropic actuators that can be used to improve the bandwidth of the control system. The anisotropic actuators are composed of piezoelectric material sandwiched between interdigitated electrodes, which enables the application of the electric field in a preferred in-plane direction. When shaped correctly the anisotropic actuators outperform traditional isotropic actuators by reducing the mismatch between the distributed actuator and point sensor at high frequencies. Testing performed on a Plexiglas panel, representative of a helicopter window, shows that the control units can increase damping at low frequencies. However high frequency performance was still limited due to the flexible boundary conditions present on the test structure.
\end{abstract}

\section{INTRODUCTION}

A helicopter powertrain generates high-frequency mechanical loads, which propagate throughout the primary structure. These loads cause vibrations in the sidewall and windows, which then radiate sound into the cabin. The resulting noise levels in the cabin can be uncomfortable for passengers.

Cabin noise in helicopters is often tonal in nature with strong tones between $500 \mathrm{~Hz}$ and $3 \mathrm{kHz}$. In this frequency range, the vibratory response of the structure is typically controlled by structural damping. As a result, manufactures often add constrained layer damping to the sidewall. Unfortunately this treatment is not effective on the windows since they need to be transparent. Fortunately other options are available for the windows. For instance, researchers have shown that embedding viscoeleastic material between layers of Plexiglas can effectively increase the structural damping without impairing visibility [1]. Another option is to use small control units installed around the perimeter of the window to generate active damping, as described in this paper.

Active damping is achieved using direct velocity feedback, and is only effective if the actuator and sensor are substantially matched. This means that the actuator and sensor have to couple to the structure in the same way. For instance, a point force actuator and point sensor constitute a matched transducer pair. Unfortunately real transducer pairs are never perfectly matched, which limits the high frequency performance of the control system. The goal of this work is to improve the bandwidth of a relatively simple active control system originally proposed by Gardonio and Elliott [2].

Gardonio and colleagues have shown that small control units, consisting of triangularly-shaped distributed actuators and point sensors, can be distributed around the perimeter of a panel to increase the structural damping [2-5]. However above 1

This material is declared a work of the U.S. Government and is not subject to copyright protection in the United States. Approved for public release; distribution is unlimited. 
approximately $1 \mathrm{kHz}$ performance is limited since the actuators do not couple to the structural response in exactly the same way as the sensors [3].

This paper focuses on the development and evaluation of a distributed anisotropic actuator designed to improve the high frequency performance of these active wedges. The new actuator couples to the panel in much the same way as a point force and therefore provides a better match with the point sensor than conventional piezoelectric actuators. This paper is divided into two parts: the first section focuses on the development and initial evaluation of the anisotropic actuator, while the second section describes the performance of an actively damped window panel designed to reduce structuralborne noise transmitted into an aircraft.

\section{TRIANGULAR ANISOTROPIC ACTUATOR}

Triangularly shaped actuators and point sensors, such as accelerometers, can couple to the structural response of a panel in the same way at low frequencies. This can be explained by representing the shaped actuator as a collection of point loads and line moments as described by Sullivan et al. [6]. Triangularly shaped actuators can be modeled using transverse point loads at each vertex and bending moments along each edge, as depicted in Fig. 1. In this case, the moment excitation along the base edge is defined as [2]

$$
m_{b}(t)=h_{s} / 2\left(e_{31}\right) v_{c}(t)
$$

where $h_{s}$ is the combined thickness of the panel and the piezoelectric patch, $e_{31}$ is a piezoelectric material constant relating the electric field applied in the 3-direction to stress induced the 1-direction, and $v_{c}(t)$ is the applied voltage. Similarly the moment excitation along the lateral edges is defined as

$$
m_{l}(t)=h_{s} / 2\left(m^{2} e_{31}+e_{32}\right) v_{c}(t)
$$

where $m=b /(2 a)$ is the slope of the lateral edge, $b$ is the base of the triangle, and $a$ is its height. The point forces generated at the base vertices are

$$
f_{b}(t)=2 m\left(h_{s} / 2\right)\left(e_{31}\right) v_{c}(t)
$$

while

$$
f_{t}(t)=(-4 m)\left(h_{s} / 2\right)\left(e_{31}\right) v_{c}(t)
$$

defines the point force at the tip of the triangular patch.

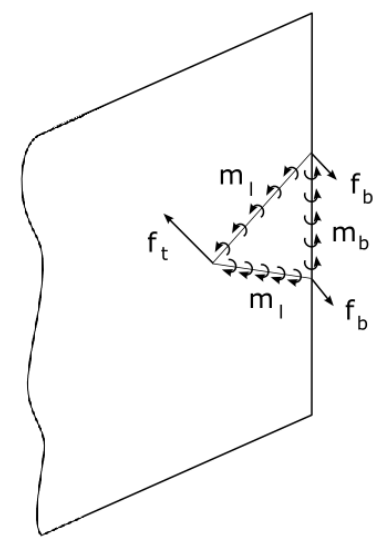

Figure 1: Triangular actuator represented in terms of point forces and line moments.

If the boundaries of the structure are clamped, then the point forces and line moments along the base of the actuator will not couple to the structural response. Therefore a single point velocity sensor placed at the vertex opposite the base edge can yield a substantially collocated frequency response (i.e. the phase will be bounded between \pm 90 degrees). However as the frequency increases and the bending wavelength approaches the dimensions of the actuator, the moments along the lateral edges couple more efficiently to the structural response than the point force at the tip of the actuator. As a result, the phase response of the open loop frequency response function tends to roll off at high frequencies. Therefore it is advantageous to eliminate the destabilizing line moments along the lateral edges of the actuator. Unfortunately this cannot be accomplished using conventional piezoelectric actuators.

Conventional actuators have uniform electrodes, which enable the application of the electric field through the thickness of the material (i.e. the 3-direction), as shown in Fig. 2 a). A positive voltage applied in the 3-direction induces equal compressive stresses in both in-plane directions (i.e. the 1- and 2 - directions). Therefore $e_{31}$ and $e_{32}$ are equal and the lateral edge moments defined in Eq. 2 cannot be eliminated. In contrast anisotropic actuators with interdigitated electrodes, as shown in Fig. 2 b), allow the application of the electric field in a preferred in-plane direction. In this case a positive voltage applied in the 1-direction induces a tensile stress in the 1-direction and a compressive stress in the 2-direction. If the triangle is shaped such that $m=\left(-e_{12} / e_{11}\right)^{\wedge} 0.5$, then the destabilizing line moments defined by Eq. 2 will equal zero.

The piezoelectric material constants for a commercially available Macro-Fiber Composite (MFC) actuator are $e_{11}=$ $11.9 \mathrm{~Pa} /(\mathrm{V} / \mathrm{m})$ and $e_{12}=-0.77 \mathrm{~Pa} /(\mathrm{V} / \mathrm{m})$. In this case the lateral edge moments equal zero when the height of the triangle is approximately twice the width of the base. In other words, the transducers should be designed to have a slope of $m=\left(-e_{12} / e_{11}\right)^{\wedge} 0.5=0.254$.

This material is declared a work of the U.S. Government and is not subject to copyright protection in the United States. Approved for public release; distribution is unlimited. 

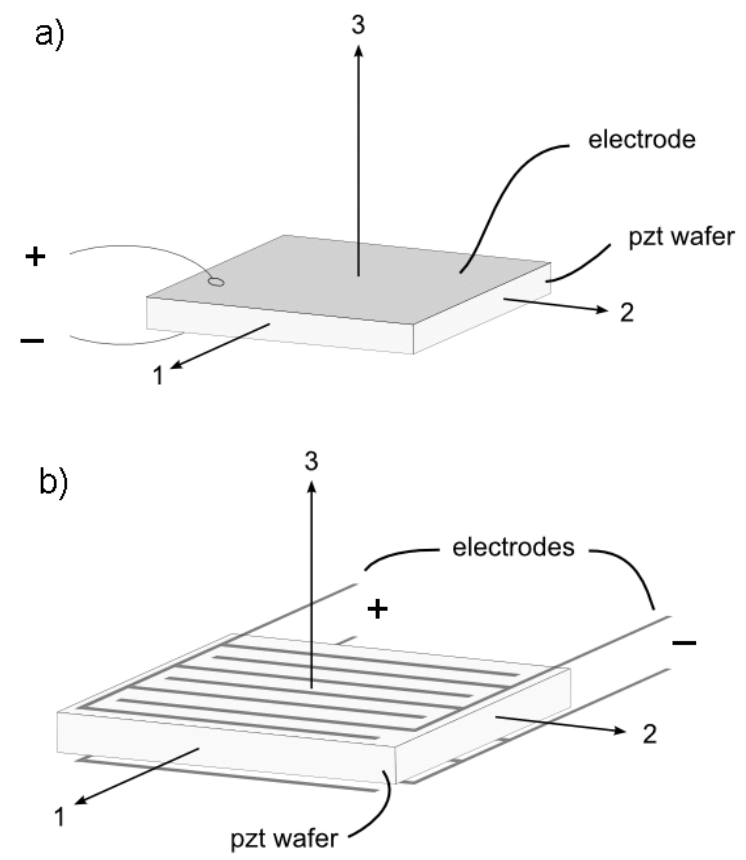

Figure 2: Sketch of (a) a conventional piezoelectric actuator, and (b) an actuator with interdigitated electrodes.

It should be noted that Gardonio et al. [4] have pointed out that modeling triangularly shaped actuators as a collection of point loads and line moments may not be appropriate in all situations. For example in the case of an equilateral triangle, the transverse point loads at each vertex should be identical, and therefore zero, due to the geometric symmetry of the actuator. However Eqs. 3 and 4 suggest that the point loads will not be equal. Since this inconsistency raises doubts concerning the accuracy of the point load / line moment formulation, the anisotropic actuator was also modeled using an elemental approach $[4,7,8]$. Figure 3 compares simulated frequency response functions of an anisotropic triangular actuator and point sensor pair and an ideal point force and point sensor pair. In this case the simulation was performed on a clamped panel, so the small differences at high frequencies are primarily due to shaping errors caused by the numerical integration routine used to simulate the response of the piezostructure. The additional mass and stiffness introduced by the piezoelectric patch also have a small impact on the highfrequency response. However in general the agreement between the two models is very good, which suggests that the new anisotropic actuator does eliminate the lateral edge moments and couples to the structure in the same way as a point force.
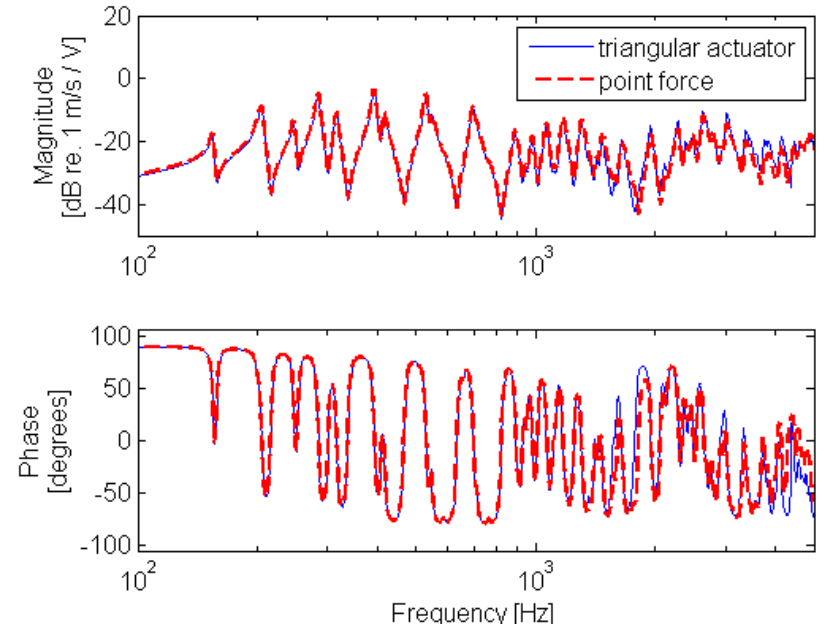

Figure 3: Frequency response functions for an anisotropic triangular actuator and point sensor pair (thin blue line), and an ideal point force input and point sensor pair (dashed red line).

After evaluating the concept numerically, two triangularly shaped actuators were fabricated. As previously described, the optimal shape is achieved by selecting the base, $b$, and height, $a$, of the triangle such that $b /(2 a)=0.254$. While the ratio is important, the overall size of the actuator is not critical and can be selected based on the application. In this case the base and height dimensions of the actuator were selected to be $3.25 \mathrm{~cm}$ (1.28 in) and $6.35 \mathrm{~cm} \mathrm{(2.5} \mathrm{in),} \mathrm{respectively.} \mathrm{The} \mathrm{only}$ difference between the two actuators was the poling direction. One actuator was poled through the thickness, which is representative of a conventional piezoelectric actuator, while the other was poled in an in-plane direction. The two actuators were mounted at the same location on opposite sides of a $1.016 \mathrm{~mm}(0.04 \mathrm{in})$ thick aluminum panel sandwiched between a $25.4 \mathrm{~mm}$ (1.0 in) thick aluminum frame. The frame was held together with fifty-two bolts torqued to $11.3 \mathrm{~N}-\mathrm{m}$ (100 in-lbs). The purpose of the frame was to create a clamped boundary around the thin aluminum panel. The triangular actuators were aligned with the boundary, as shown in Fig. 4, and an accelerometer was placed at their tip. This test structure was relatively small, with exposed panel dimensions of $35.6 \mathrm{~cm}$ (14 in) by $25.4 \mathrm{~cm}$ (10 in).

This material is declared a work of the U.S. Government and is not subject to copyright protection in the United States. Approved for public release; distribution is unlimited. 


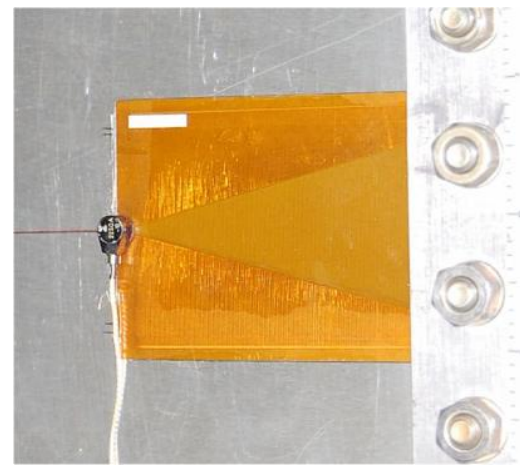

Figure 4: Triangularly shaped anisotropic actuator mounted along the perimeter of the aluminum panel.

Frequency response functions were then acquired between each actuator and sensor. In this case the sensed variable was the integrated response from the accelerometer (velocity). Figure 5 shows the Nyquist diagram of the frequency response functions through $3 \mathrm{kHz}$. The thin dashed black line corresponds to the conventional actuator and the solid red line corresponds to the anisotropic actuator. The phase response for matched transducer pairs will be bounded between \pm 90 degrees and therefore the response will be strictly positive real. In other words the Nyquist diagram will be restricted to the right-half plane if the transducers are matched. As shown in the figure, the conventional actuator has relatively large loops in the left half plane, while the anisotropic actuator is almost completely positive real. This demonstrates that the anisotropic actuator is coupling to the structure in essentially the same way as the point sensor in this frequency range. The small loops in the left half plane are probably due to shaping errors, accelerometer placement errors, or frame dynamics (resulting in a nonclamped boundary).

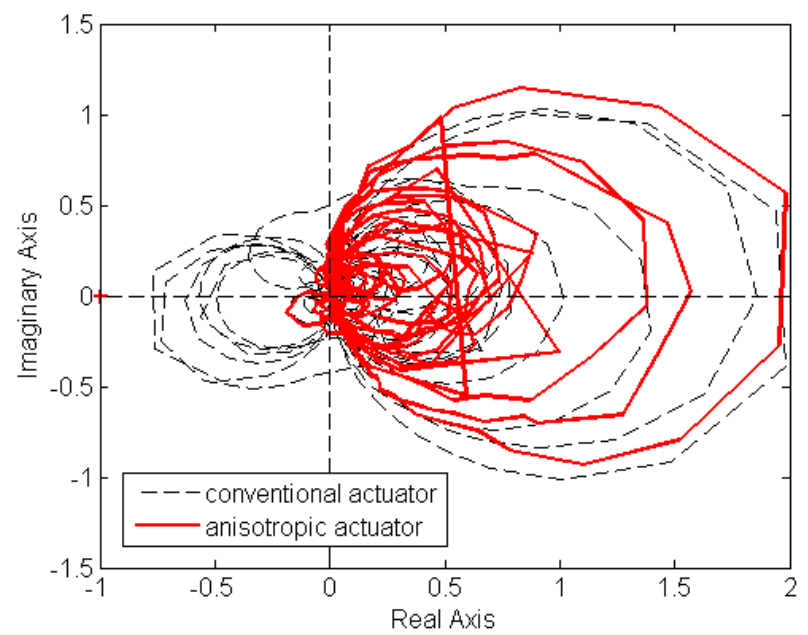

Figure 5: Nyquist diagram of the open loop frequency response function for a conventional actuator and point sensor (dashed black line) and an anisotropic actuator and point sensor (solid red line) between $2.5 \mathrm{~Hz}$ and $3 \mathrm{kHz}$.
These results are promising and suggest that the lateral edge moments can be eliminated by appropriately shaping anisotropic actuators. This has significant implications for active control systems that rely on direct velocity feedback. The Nyquist diagram in Fig. 5 suggests that a control unit consisting of a conventional actuator and point sensor would have a $2.3 \mathrm{~dB}$ gain margin (through $3 \mathrm{kHz}$ ) while an anisotropic actuator and point sensor pair would have a gain margin of $17.9 \mathrm{~dB}$. Therefore the control gain on the system with the anisotropic actuator could be increased by a factor of 6 relative to the conventional actuator, which could result in significantly better closed loop performance. Following the initial evaluation of the shaped anisotropic actuator, subsequent work focused on designing, building, and testing 16 control units that could be used around the perimeter of a larger helicopter window.

\section{TEST SETUP}

This section describes the control units and the test setup used to evaluate the active control system. Each control unit consists of a control circuit, power amplifier, triangularly shaped actuator, accelerometer, and signal conditioner, as shown in Fig. 6. While all the components are relatively compact, the size and weight of the electronics could be reduced further. Miniaturizing the electronics was not a priority in this project, but it would make the control system more practical.

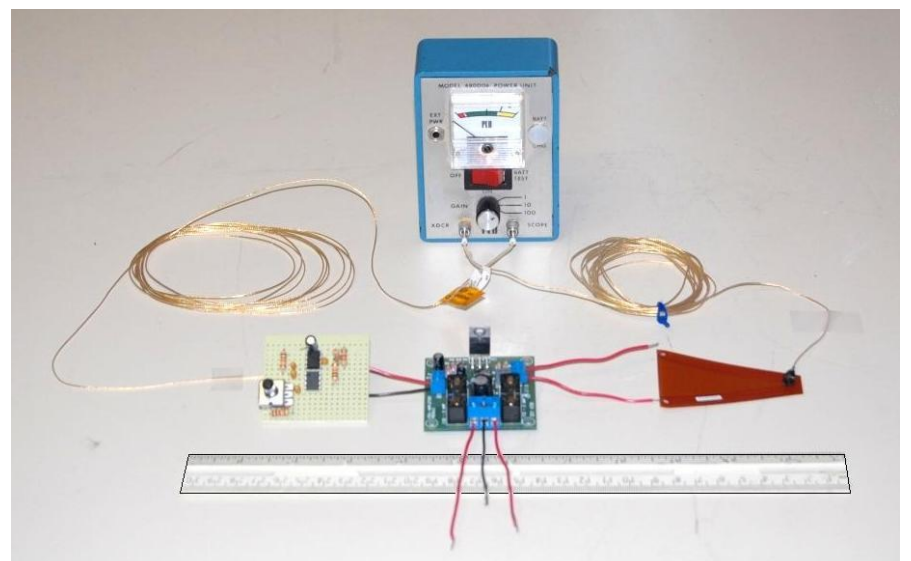

Figure 6: Each control unit consists of a control circuit (left), power amplifier (middle front), actuator (right), accelerometer (on top of the actuator), and signal conditioner (middle back). The ruler in the foreground is $30.5 \mathrm{~cm}(12 \mathrm{in})$ long.

The control circuit consists of three main parts, as depicted in Fig. 7. A $2^{\text {nd }}$ order Bessel high-pass filter is located at the circuit input. This filter is used to attenuate low-frequency signals before they are integrated. A $100 \mu \mathrm{F}$ capacitor is also placed before the integrator to remove any DC bias from the signal. An integrator is used to generate a signal proportional to velocity instead of acceleration. Finally an adjustable gain amplifier is implemented with a potentiometer that enables gain adjustments. All results presented in this paper were acquired

4

This material is declared a work of the U.S. Government and is not subject to copyright protection in the United States. Approved for public release; distribution is unlimited. 
with the potentiometer set to $17 \mathrm{k} \Omega$. The capacitor and resistor included on the circuit output function as a passive high pass filter, which was necessary to remove low frequency oscillations caused by the op-amps.

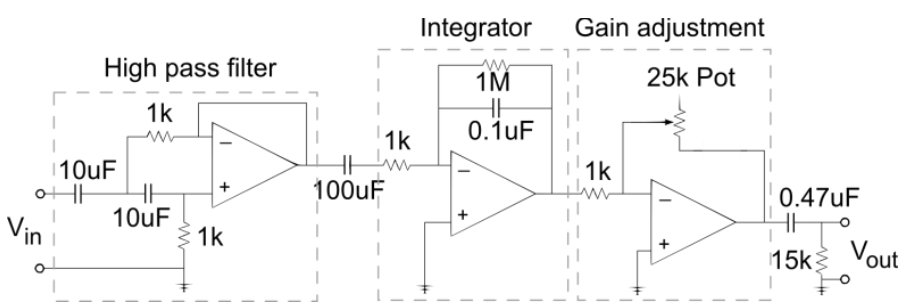

Figure 7: Diagram of the analog control circuit.

The power amplifiers were built from $25 \mathrm{~W}$ high fidelity audio amplifier kits. These amplifiers are inexpensive and relatively compact with a footprint of 53 × $50 \mathrm{~mm}(2.1 \times 2.0$ in). All 16 amplifiers were powered by 4 Datel BCM-15/200 dual $\pm 15 \mathrm{~V}$ power supplies. Even though the amplifiers are rated at $25 \mathrm{~W}$, the power draw from each amplifier during closed-loop operation was only $1.5 \mathrm{~W}$.

New anisotropic actuators were fabricated with the same effective dimensions as the prototype shown in Fig. 4. However the footprint of the new actuators was reduced by reshaping the interdigitated electrode pattern and by removing extra Kapton surrounding the actuator. The sensors consist of miniature accelerometers. These accelerometers are small and lightweight $(0.4 \mathrm{gm})$ with a very wide bandwidth $(2 \mathrm{~Hz}$ to $15 \mathrm{kHz}$ ).

Although a handheld signal conditioner is pictured in Fig. 6, a larger 16 channel signal conditioner was used during the test. The 16 channel signal conditioner has continuous gain adjustment from 0.1 to 200 and built-in fourth-order Butterworth low pass filters. For these tests, the cutoff frequency on the filters was maxed out at $20 \mathrm{kHz}$. The adjustable gain provided a convenient way to tune the gain of each control unit without adjusting the potentiometer in the control circuit.

The 16 control units were evaluated on a relatively large Plexiglas window panel, representative of a helicopter window. The panel is $4.45 \mathrm{~mm}(0.175 \mathrm{in})$ thick and is sandwiched between a $38.1 \mathrm{~mm}$ (1.5 in) thick aluminum frame. The frame is held together with fifty bolts torqued to $16.9 \mathrm{~N}-\mathrm{m}$ (150 inlbs). The outer dimensions of the frame are $90.4 \mathrm{~cm}$ (35.6 in) by $70.9 \mathrm{~cm}$ (27.9 in), while the nominal dimensions of the window are $45.5 \mathrm{~cm}$ (17.9 in) by $65.0 \mathrm{~cm}$ (25.6 in). The 16 transducer pairs were installed around the perimeter of the window panel as shown in Fig. 8. The structure was excited by a single electromagnetic shaker suspended from an adjustable excitation stand. The shaker was attached to the structure using a $6.3 \mathrm{~cm}$ (2.5 in) long flexible stinger and an impedance head.

A Polytec PSV-300 scanning laser vibrometer was positioned $3.50 \mathrm{~m}$ (137.6 in) from the Plexiglas window and was aligned with the $17 \times 25$ measurement grid shown in Fig. 8. The grid consists of $6.35 \mathrm{~mm}(0.25 \mathrm{in})$ diameter circular pieces of reflective tape, which were bonded to the panel. The reflective tape was needed to measure the vibration of the transparent window using the laser.

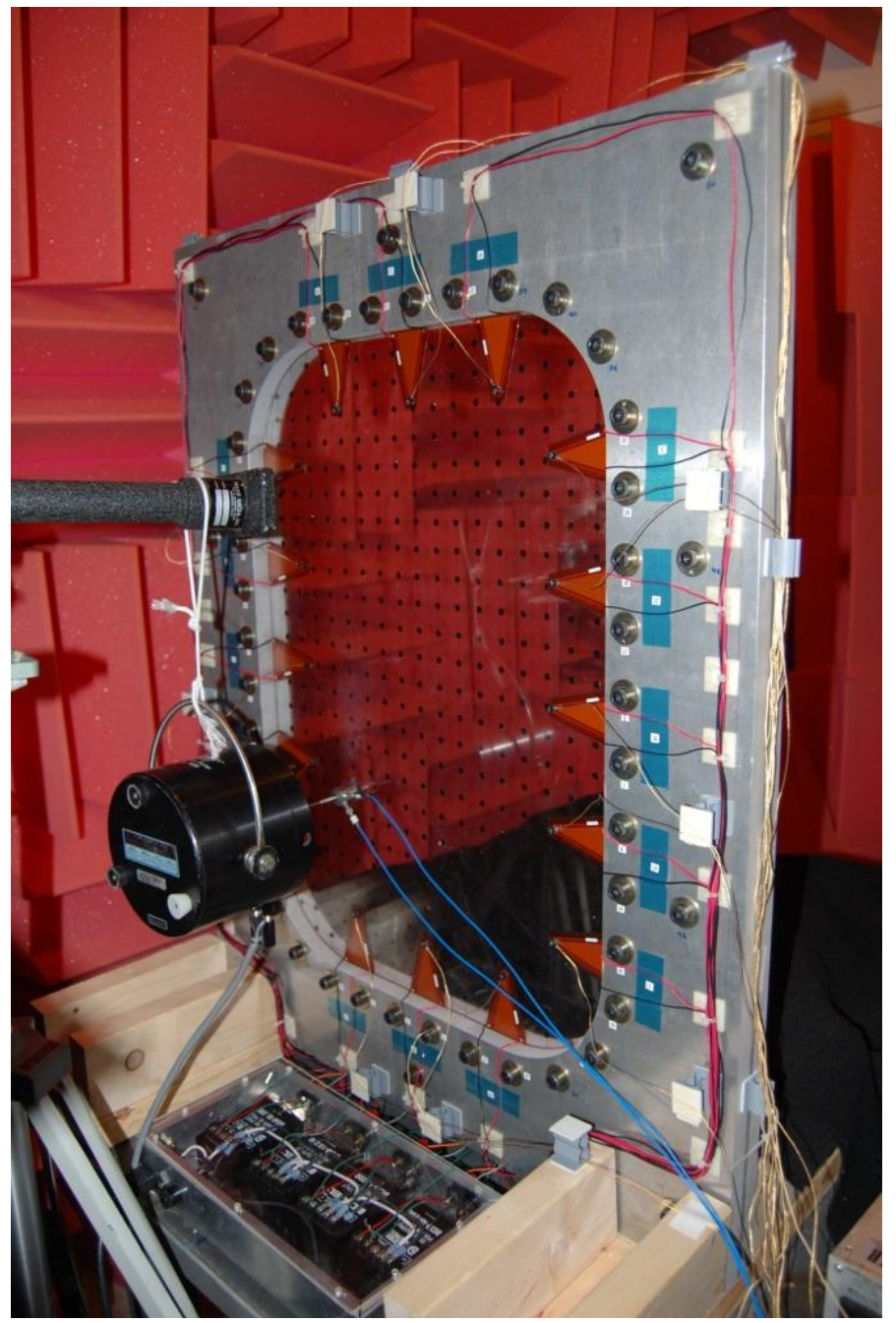

Figure 8: Photograph of the test setup.

\section{ACTIVE DAMPING OF THE PLEXIGLAS WINDOW}

This section describes the performance of the control system installed on a Plexiglas window panel. Initial measurements of the open-loop frequency response function for a single control unit are shown in Fig. 9. The FRF was acquired by driving one of the audio amplifiers with a broadband random signal while measuring the output of the corresponding control circuit. Therefore the FRF includes the structural dynamics as well as the high frequency roll-off due to the low-pass filter in the signal conditioner (around $20 \mathrm{kHz}$ ). Since the control circuit includes an integrator, the output is proportional to the velocity at the tip of the actuator. There are a few things to notice in the FRF. First the transducer pair efficiently couples to the low order structural modes and the phase is bounded at low frequencies. Unfortunately the phase response begins to roll off at $600 \mathrm{~Hz}$. Recall that when the transducers were tested on the small aluminum panel, the phase 5

This material is declared a work of the U.S. Government and is not subject to copyright protection in the United States. Approved for public release; distribution is unlimited. 
response was essentially bounded through $3 \mathrm{kHz}$. The nonminimum phase behavior observed on this structure could be due to actuator shaping errors, accelerometer placement errors, or frame dynamics. Since the primary difference between this test and earlier tests was the structure, the phase roll off observed on the Plexiglas panel was attributed to frame dynamics. When the boundaries are flexible, the bending moments around the base of the actuator can couple to the structural response more efficiently than the point force at the tip.
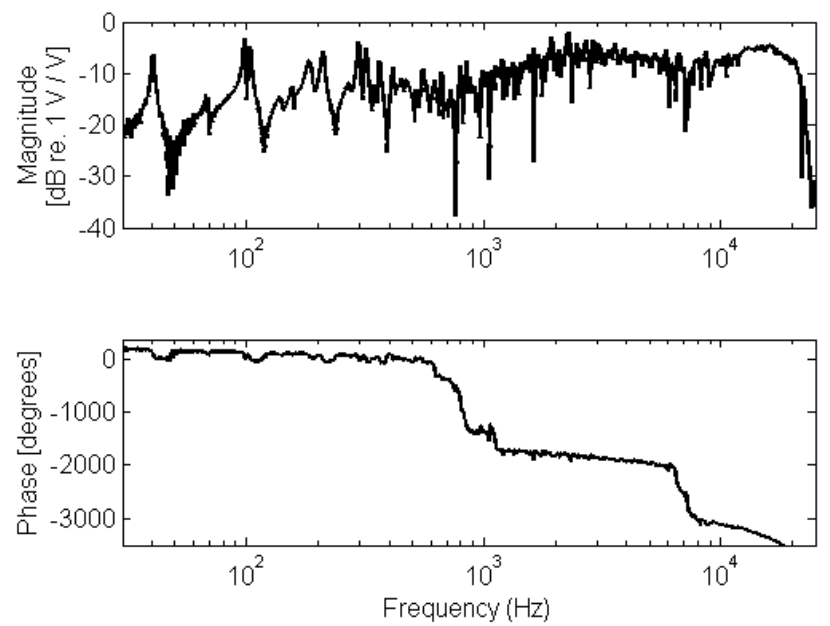

Figure 9: Open loop frequency response function for one control unit.

Figure 10 shows the Nyquist diagram of the open loop FRF shown in Fig. 9. The stability of a single control unit can be inferred from the Nyquist stability criterion. If the plant and controller are both stable, then the Nyquist stability definition states that the closed loop system will be stable if and only if the polar plot of the open-loop frequency response does not encircle the $(-1,0)$ point as the frequency varies from $-\infty$ to $\infty$ [9]. Based on the Nyquist diagram, it is clear that this control unit will be stable when the loop is closed. However just because each individual control loop is stable, does not mean that all 16 control units will be stable when implemented together. To evaluate stability of the entire set of control loops, the generalized Nyquist stability criterion can be used [9]. If the plant and individual controllers are stable, then the multichannel system will be stable as long as the locus of the determinant of $(I+G H)$ does not encircle $(0,0 j)$ as the frequency varies from $-\infty$ to $\infty$. In this case $G$ is a $16 \times 16$ matrix of FRFs, and $H$ is diagonal matrix with the feedback gains along the main diagonal.

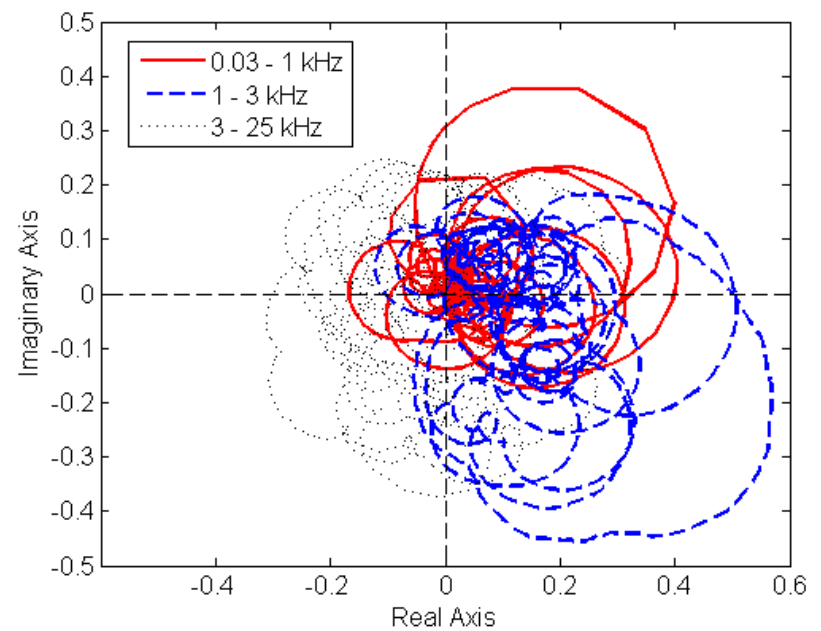

Figure 10: Nyquist diagram of the open loop FRF for one control unit between $30 \mathrm{~Hz}$ and $1 \mathrm{kHz}$ (solid red line), between $1 \mathrm{kHz}$ and $3 \mathrm{kHz}$ (dashed blue line), and between $3 \mathrm{kHz}$ and $25 \mathrm{kHz}$ (dotted black line).

The maximum control gains for the multichannel system will always be less than or equal to the maximum control gains for the individual loops. One of the advantages of direct velocity feedback is that it does not require a complicated system model for control. So instead of acquiring 256 FRFs and evaluating the generalized Nyquist criterion, a single nominal control gain was selected for all 16 control units. The gain of all units was then slowly increased until the combined system went unstable, which was identified by a ringing denoting a limit cycle response. The gain was then reduced to the maximum stable value. In practice it would be advisable to reduce the gain by some amount (e.g. a factor of 2), to obtain adequate stability margins, however the maximum stable gain was used for this evaluation.

The performance of the control system was evaluated in terms of the spatially averaged velocity per unit force as shown in Fig. 11 and 12. The control system reduces individual resonance peaks by as much as $11 \mathrm{~dB}$ and achieves a $3 \mathrm{~dB}$ integrated reduction through $550 \mathrm{~Hz}$. However above $600 \mathrm{~Hz}$, control performance is negligible. Since the phase response for the FRFs is not bounded between \pm 90 degrees, high-frequency spillover is unavoidable. In this context spillover means the magnitude of the closed-loop response is greater than the openloop response.

This material is declared a work of the U.S. Government and is not subject to copyright protection in the United States. Approved for public release; distribution is unlimited. 


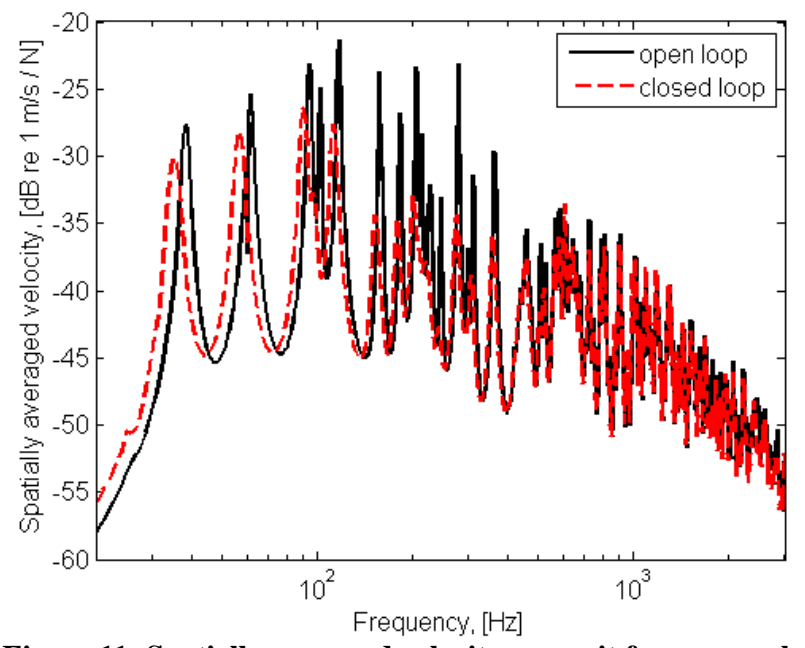

Figure 11: Spatially averaged velocity per unit force: open loop (solid black line), and close loop (dashed red line).

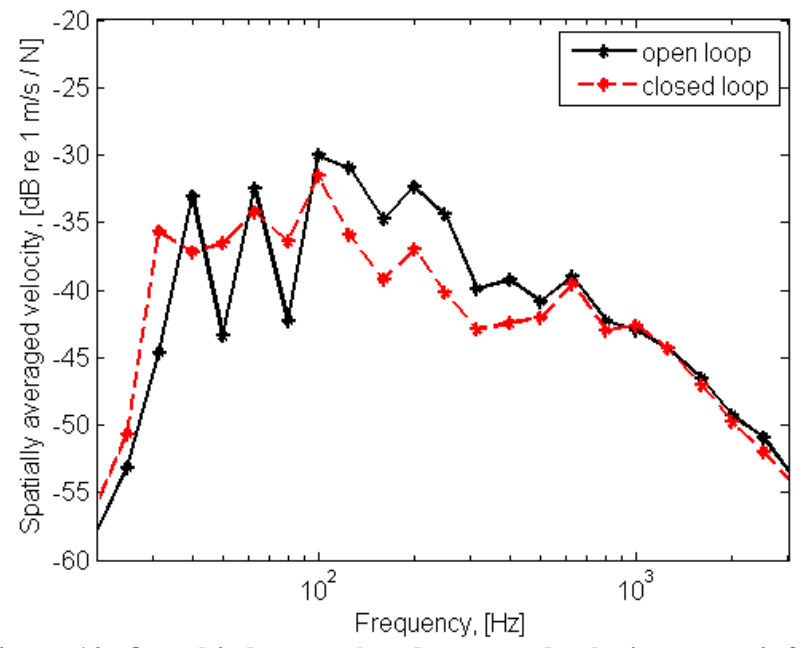

Figure 12: One-third octave band averaged velocity per unit force: open loop (solid black line), and closed loop (dashed red line).

As shown in Fig. 11, the control system not only reduces the amplitude of the low frequency peaks, but it also shifts them to the left. This behavior is caused by the low frequency phase introduced by the high-pass filters. Recall that two highpass filters were included in the control circuits. With cutoff frequencies around $20 \mathrm{~Hz}$, the filters add significant phase through $100 \mathrm{~Hz}$, as shown in Fig. 13. The thin blue lines are included in the figure to indicate \pm 90 degrees. The additional low-frequency phase rotates the loops of the Nyquist diagram counterclockwise resulting in spillover below the resonance frequencies. The net result is a shift in the closed loop peaks to the left and an overall reduction of control performance at lower frequencies.
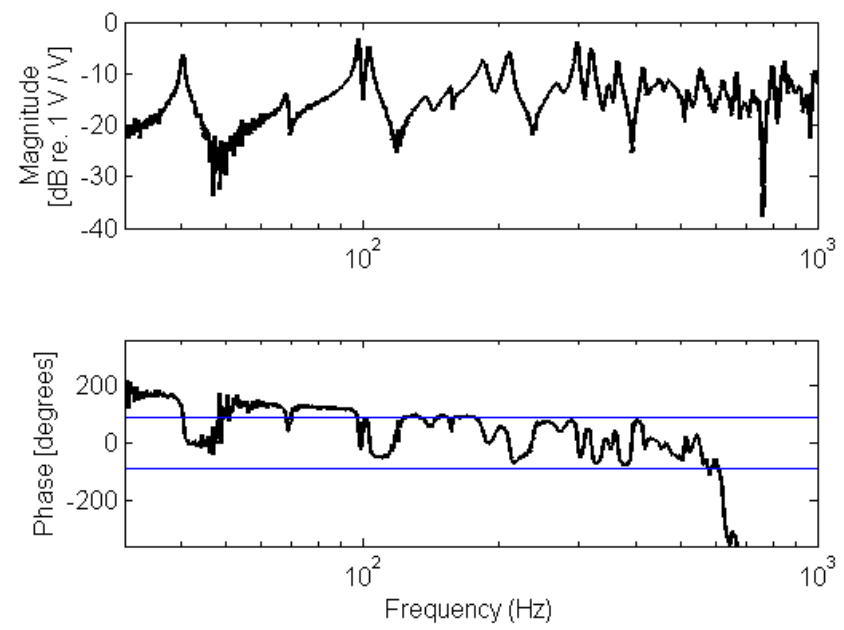

Figure 13: Open loop frequency response function from $30 \mathrm{~Hz}$ to $1 \mathrm{kHz}$ for a single control unit. The blue lines indicate $\pm \mathbf{9 0}$ degrees.

\section{CONCLUDING REMARKS}

This paper has described the development and evaluation of a distributed anisotropic actuator, which was designed to improve active damping. In preliminary tests, the anisotropic actuator was shown to couple to the structural response of a clamped aluminum panel in much the same way as a point sensor through $3 \mathrm{kHz}$. However when the control units were installed around the perimeter of a larger Plexiglas window panel, the control system's bandwidth was limited to $600 \mathrm{~Hz}$. Flexible boundaries are postulated to be the cause for the limited bandwidth, which constitutes a serious practical limitation of this approach. When the boundaries are flexible, the bending moments around the base of the actuator will couple to the structural response more efficiently than the point force at the tip at high frequencies. Future work will focus on reducing the control system's sensitivity to the boundaries by increasing the amplitude of the tip force relative to the bending moment at the base. To simultaneously accomplish this and eliminate the lateral edge moments, the piezoelectric material constants will have to be modified.

Despite the limitations imposed by the flexible boundary, the control system still reduced the spatially averaged velocity of the panel by $11 \mathrm{~dB}$ at individual resonance peaks and achieved an integrated reduction of $3 \mathrm{~dB}$ through $550 \mathrm{~Hz}$. Although this system may not be effective for helicopters in its current form, it could find use on windows in other transportation vehicles with lower frequency structure-borne noise.

\section{REFERENCES}

[1] Buehrle, R. D., Klos, J., and Gibbs, G. P., 2004, "Damped windows for aircraft interior noise control," Proc. Noise-Con 2004, Baltimore, Maryland.

[2] Gardonio, P., and Elliott, S. J., 2005, "Smart panels with

7

This material is declared a work of the U.S. Government and is not subject to copyright protection in the United States. Approved for public release; distribution is unlimited. 
velocity feedback control systems using triangularly shaped strain actuators," J. Acoustical Society of America, 117(4), pp. 2046-2064.

[3] Aoki, Y., Gardonio, P., and Elliott, S. J., 2008, "Rectangular plate with velocity feedback loops using triangularly shaped piezoceramic actuators: Experimental control performance," 123(3), pp. 1421-1426.

[4] Gardonio, P., Aoki, Y., and Elliott, S. J., 2009, "Stability and control performance of decentralized velocity feedback loops using triangular actuators," Proc. Active 2009, Ottawa, Canada. [5] Gardonio, P., Bianchi, E., and Elliott, S. J., 2004, "Smart panel with multiple decentralized units for the control of sound transmission. Part I: theoretical predictions," J. Sound and Vibration, 274, pp. 163-192.

[6] Sullivan, J. M., Hubbard, J. E., and Burke, S. E., 1996, "Modeling approach for two-dimensional distributed transducers of arbitrary spatial distribution," J. Acoustical Society of America, 99(5), pp. 2965-2974.

[7] Clark, R. L., Saunders W. R., and Gibbs G. P., 1998, Adaptive Structures, Dynamics and Control. John Wiley and Sons, NY, Chap 5.

[8] Schiller, N. H., 2007, "Decentralized control of sound radiation from periodically stiffened panels," $\mathrm{PhD}$ thesis, Virginia Tech, Blacksburg, VA.

[9] Elliott, S. J., 2001, Signal Processing for Active Control, Academic Press, London, UK, Chap. 6.

This material is declared a work of the U.S. Government and is not subject to copyright protection in the United States. Approved for public release; distribution is unlimited. 